\title{
BACKANALYSIS OF MEASURED MOVEMENTS IN AGEING TUNNELS
}

\author{
C. de Santos. ${ }^{1}$, A. Ledesma* ${ }^{2}$, A. Gens. $*^{3}$ \\ *Department of Geotechnical Engineering and Geosciences ${ }^{1}$ e-mail: cristian.desantos@upc.edu, \\ 2e-mail: alberto.ledesma@upc.edu, ${ }^{3}$ e-mail: antonio.gens@upc.edu
}

Keywords: Inverse analysis, tunnels, ageing, Plaxis

\begin{abstract}
The paper describes a procedure to perform backanalysis in a systematic manner in the context of ageing tunnels. Basically the input data include information on relative displacements or deformations of specific points in the lining of a tunnel exhibiting long term movements and the objective of the procedure is to identify a set of parameters that characterize the surrounding soil and the lining properties. Any damage in the infrastructure should give a change in those parameters identified. The analysis should be able to obtain useful information for the manager of the tunnel, in terms of maintenance, repair of some sections, warning in case of danger, etc. The paper presents the basic theory of parameter identification and an application to the backanalysis of a tunnel section from London Underground, where some measurements of rotation of lining segments are available.
\end{abstract}

\section{INTRODUCTION}

The work presented in this paper has been developed in the context of the European Science Foundation Project "Micro-Measurement and Monitoring System for Ageing Underground Infrastructures". The research project focus on the monitoring of the deterioration of underground structures by means of new technologies based on wireless sensor networks, including computer vision and miniature electromechanical sensors. Ageing of tunnels is observed as a deterioration of the lining in most cases. Opening of cracks, strains or relative displacements or rotations between two points constitute typically the main variables that can be measured on the lining surface. Other basic measurements like pore water pressures in the surrounding soil or absolute displacements could be considered as well in further developments.

All the data provided by these sensors must be gathered and stored in a comprehensive database. Due to the large amount of data involved, a postprocessor is required, in order to provide to the manager responsible for the maintenance a first insight into the overall behaviour of the infrastructure.

After identifying the most critical sections, it is necessary to perform a more sophisticated analysis in order to relate the damage with a cause. If the reason for that damage is not found out, it will be difficult to make appropriate decisions. This is often a difficult task due to the nature of the failure modes, involving complicated interactions between the underground structures and the adjacent ground. Therefore, it is necessary to analyze the mechanical response of the underground structure and the surrounding soil. There are several commercial codes based on the finite element method that can assist in that task. They usually solve the problem in a direct manner; that is, given a particular geometry and a set of parameters, they predict the stresses and strains of the structure. However, in this case field measurements constitute the input data, and the manager of the system would expect an assessment of the 
integrity and properties of the materials involved. This is in fact an inverse problem, in which mechanical parameters are estimated from measurements as strains or relative displacements.

The estimation of soil and rock parameters based on field instrumentation data is a traditional and a common procedure in Geomechanics. However, the use of optimization techniques to perform backanalysis in a more systematic manner is a relatively recent development (Gioda \& Sakurai, 1987). From a mathematical point of view, the identification of parameters results in a minimization problem. Nowadays, the use of system identification and optimization techniques allows the performance of this type of analyses in a more rational and objective manner. Examples of backanalysis in the context of tunnel excavation problems are described in Ledesma et al $(1996,1997)$ and Gens et al $(1988,1996)$ among others. In those works, a maximum likelihood approach is presented to estimate the required parameters. The main difficulty when estimating parameters is that several combinations of them may give similar results in terms of measurement variables and therefore the solution is not unique. Because of that, part of the research in this field has been devoted to the improvement of the optimization process itself.

This paper presents an example of backanalysis performed using field data from an old tunnel in the context of the research project mentioned above. First, a basic theory about parameter identification is presented. Then, a real case involving a tunnel section in London Underground is described. In this case, ageing is due to consolidation and drainage of the surrounding soil. The Ko parameter and the stiffness of the joints between lining segments have been identified. In fact, both parameters are very difficult to measure and this type of techniques could be very useful in practice.

\section{BACKANALYSIS PROCEDURE}

It is assumed that a fixed and deterministic model relates a set of variables $\mathbf{x}$ and a set of parameters $\mathbf{p}$. Some of the variables $\mathbf{x}$ are measured and form the vector of measurements $\mathbf{x}^{*}$. The best parameters are those that minimize the difference between measured and computed variables. A simple procedure to establish that, is defining the so called "objective function", F:

$$
F=\sum_{i=1}^{m}\left(x_{i}^{i}-x_{i}\right)^{2}
$$

where $m$ is the number of measurements. $F$ represents the error between the measurements and the same variables computed with the model, computed using a least squares criterion. Expression (1) can be generalized when measurements are not independent or have different errors (Ledesma et al, 1996). Note that $F$ is a function of the parameters, as $\mathbf{x}=\mathrm{M}(\mathbf{p})$, where $\mathrm{M}$ represents the model. Minimizing $F$ will provide with the set of parameters that best simulate the measurements obtained.

It should be pointed out that $F$ depends in a nonlinear manner on the parameters. The model $\mathrm{M}$ is usually represented by a Finite Element analysis. Even when a linear law is used, the objective function, $F$, is nonlinear with respect to the parameters $\mathbf{p}$. That makes difficult to find the minimum of $F$. When only one or two parameters are identified, a simple inspection of the values of $F$ may be enough to estimate the minimum. This has been the case in the example described in this paper. Moreover, the plot of the objective function is useful to understand the sensitivity of measurements to the parameters considered in the analysis. However, if more than two parameters are involved, then it is more convenient to use any suitable minimization algorithm.

In general it is necessary to choose between methods that only need evaluations of the function and methods that also require computations of the derivative of that function. Algorithms using the derivative of the objective function are expected to be more powerful 
than those using only the values of the function itself, but they may be generally more time consuming. A combination of both approaches can be also a good strategy in complex problems.

\section{APLICATION TO AGEING TUNNELS IN LONDON}

\subsection{Site description}

The application of the backanalysis procedure outlined above refers to a particular section in the Northbound tunnel of the Jubilee Line, between Baker Street and Bond Street in London Underground, built in the seventies using a shield.

In the simulation, both tunnels have been taken into account, although only measurements from the Northbound tunnel have been considered in the backanalysis procedure. This is because ageing on those tunnels is apparently due to the consolidation and drainage of the surrounding soil and, therefore, both tunnels must be considered in the geometry for the analysis.

The Northbound tunnel has a diameter of about $4 \mathrm{~m}$ and was excavated mainly in London clay. The lining is made of 22 concrete segments in most of the tunnel.

\subsection{Measurements available}

Measurements available refer to the rotation between segments due to the movements of the lining. Probably the initial shape of the lining when constructed was not circular (Lyons, 1979) but it is difficult to distinguish between original imperfections and movements produced along time, as only one set of displacements has been measured and compared with the theoretical circular shape.

The measurements available give the rotation between two contiguous segments. That rotation originates a displacement of the contact point between segments, which is known as "eccentricity". Values of "eccentricity" for all the joints have been used as main data in the identification process.

\subsection{Identification of parameters}

The direct problem has been analyzed by using the Finite Element Code "Plaxis" (version 8 in 2D). Figure 1 shows the finite element mesh used in the simulations. As this analysis is focused on the inverse problem, a simple constitutive law was selected for the direct problem. In this case, an elastic - perfect plastic law based on Mohr-Coulomb and available in Plaxis was used. First, the initial stresses are generated assuming a Ko parameter in advance. Then the tunnel is excavated, installing the lining and computing for equilibrium after release of the initial stresses. We have used an additional contraction loss of the lining corresponding to a volume loss of $1.4 \%$, in order to take into account the effect of the shield (gaps, open excavation, installation of segments, etc), just during construction. That value is just an estimation based on experience. After that state, drainage is allowed, and a fully drained analysis is performed, keeping constant the water table and the hydraulic conditions in the geometry. Future improvements of the model will include the consideration of the actual groundwater history in the zone.

For the inverse problem we have considered the Ko parameter of London clay and the stiffness of the joints as the main parameters to be identified. On the one hand, the stiffness of the segment joints with respect to rotation is a difficult parameter to know in advance and therefore a backanalysis constitutes the only manner to obtain a consistent value. On the other hand, the Ko parameter, although well known for London clay, has an important influence on the deformed shape of the linings.

The objective function adopted for this case, F, had the following expression: 


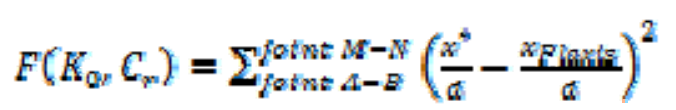

Where $\mathrm{x}_{\text {plaxis }}$ refers to the eccentricity values obtained from the direct analysis using Plaxis and $x^{*}$ corresponds to the measured eccentricities. Also, " $d$ " is the segment thickness (used to make the error dimensionless) and $\mathrm{Cr}$ the joint stiffness for rotation.

The analysis was performed considering both, $\mathrm{Cr}$ and Ko, as parameters to be identified. The combinations of $\mathrm{Cr}$ and Ko were used to solve the direct problem, and then the objective function (2) was computed. In this manner it was possible to plot that function and to obtain the minimum by direct inspection also. That is, a minimization algorithm was not required in this problem. Figure 1 presents that function for this case. The minimum is found for a value of $\mathrm{Cr}$ close to $10000 \mathrm{kNm} / \mathrm{rad} / \mathrm{m}$ and a value of $\mathrm{Ko}=1.5$. This value for the earth pressure coefficient at rest is quite common in many Finite Element analyses involving London clay.

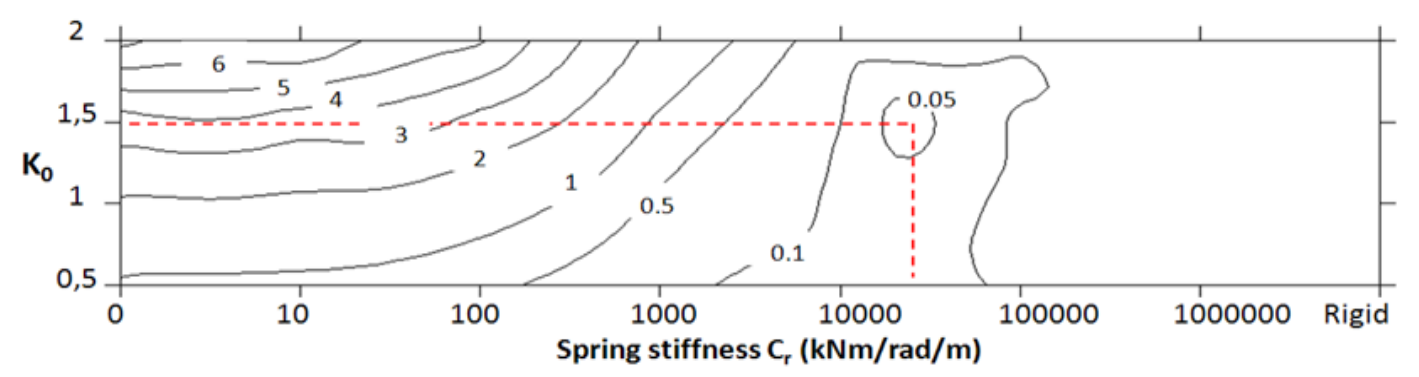

Figure 1. Objective function $\mathrm{F}\left(\mathrm{C}_{\mathrm{r}}, \mathrm{K}_{0}\right)$

\section{CONCLUSIONS}

The paper presents an example of estimation of parameters from measurements from the lining of ageing tunnels. This process is expected to be performed in an automatic manner in order to assess the evolution of the lining of tunnels undergoing deterioration. Inverse problem techniques can be used in this process as they have proven to be efficient in Geomechanical problems. The identification of Ko and the joint stiffness of a segmental lining has been identified from measurements of rotations between segments in a real case involving a tunnel in London Underground. Joint stiffness is particularly difficult to measure in practice and may be related to lining deterioration. In this respect, a systematic application of the identification of parameters techniques, constitute a useful tool for the safety assessment of old tunnels.

\section{REFERENCES}

[1] Gens, A., Ledesma, A., Alonso, E.E. (1988). Back analysis using prior information Application to the staged excavation of a cavern in rock. Numerical Methods in Geomechanics, Swoboda ed., Innsbruck, Balkema, p. 2009-2016.

[2] Gens, A., Ledesma, A., Alonso, E.E. (1996). Estimation of parameters in Geotechnical Backanalysis - II. Application to a tunnel excavation problem. Computers and Geotechnics, 18:29-46

[3] Gioda, G., Sakurai, S. (1987). Back analysis procedures for the interpretation of field measurements in Geomechanics. Int. J. for Numerical Analyt. Methods Geomech., 11:555583.

[4] Ledesma, A., Gens, A., Alonso, E.E. (1996). Estimation of parameters in Geotechnical Backanalysis - I. Maximum Likelihood Approach. Computers and Geotechnics, 18:1-27.

[5] Ledesma, A., Romero, E. (1997). Systematic backanalysis in tunnel excavation problems as a monitoring technique. Proc. $14^{\text {th }}$ Int. Conf. Soil Mech. Found. Engng. Hamburg, Balkema, 3, p.1425-1428. 